\title{
Wormlike reverse micelles in lecithin/bile salt/water mixtures in oil
}

\author{
Jacopo Cautela ${ }^{\mathrm{a}}$, Mauro Giustini ${ }^{\mathrm{a}}$, Nicolae Viorel Pavel $^{\mathrm{a}}$, Gerardo Palazzo ${ }^{\mathrm{b}}$, Luciano Galantini ${ }^{\mathrm{a}, *}$ \\ a Department of Chemistry, Sapienza University of Rome, P. le A. Moro 5, 00185 Rome, Italy \\ b Department of Chemistry, University of Bari, via Orabona 4, 70126 Bari, Italy
}

A R T I C L E I N F O

\section{Keywords:}

Wormlike micelles

Lecithin

Rheology

SAXS

Bile salts

\begin{abstract}
A B S T R A C T
Knowing the ability of water and bile salts to promote the reverse wormlike micelle growth in lecithin/water or lecithin/bile salt mixtures in oil, this work was aimed at elucidating the association properties of the three solutes lecithin, water and the bile salt (BS) sodium deoxycholate in cyclohexane. By systematically changing the fraction of the two additives (i.e.: water and BS) we could identify a region at low additive/lecithin molar ratios where stable wormlike micelle dispersions were formed. Small angle X-ray scattering and oscillatory rheology measurements demonstrated that the ability of bile salt and water to transform the originally spherical lecithin reverse micelles into wormlike micelles and thereby impart to the sample viscoelastic properties is preserved in the three-solute mixture. The results suggest that reverse micelle including both bile salt and water are formed in this system. Reasonably the two primers interact with the same region of the lecithin headgroups and are complementary in altering the packing parameter of the amphiphile to values suitable for the formation of cylindrical aggregates.
\end{abstract}

\section{Introduction}

Wormlike micelles, formed by very long cylindrical and flexible aggregates of different amphiphiles, have been extensively investigated in the past several decades because of their remarkable visco-elastic properties that are exploited in numerous technological fields. The interest of these systems is particularly relevant when they are formed by biological surfactants in the light of their potential employment in biochemical and biomedical applications. Phospholipids and bile salts represent two important types of biological surfactants particularly prone to form mixed aggregates with different structures [1-4]. Bile salts (BSs) are anionic natural steroidal surfactants found predominantly in the bile of mammals (humans included) and other vertebrates and deeply involved in various biological processes $[5,6]$. The amphiphilic structure of these molecules is quite different from that of conventional head-tail surfactants and for this reason they manifest some peculiarities in their self-assembly behavior [7-12]. In fact, beside the negatively charged head, they exhibit a rigid steroidal structure with a variable number of hydroxyl groups in specific positions (Fig. 1).

The BSs are synthesized in the liver using cholesterol as starting material and act as solubilizers and emulsifiers of non-polar material, in particular cholesterol itself, lipids, fat-soluble vitamins, fatty acids and monoglycerides $[5,6]$. Moreover, they are widely used as starting materials in the preparation of synthetic derivatives by changing their amphiphilic structure and by introducing specific functionalities
$[9,13,14]$. Due to the steroid rigidity and the peculiar distribution of hydrophobic and hydrophilic domains, these molecules are particularly attractive for the bottom up construction of complex nanostructures. They often self-assemble in tubes or fibers and behave as low molecular weight gelators [15-26]. The tubes have cross section diameters spanning a wide range of values $(3-450 \mathrm{~nm})[9,19,27-34]$ and sometimes they form through appealing $\mathrm{pH}[35,36]$ or temperature responsive aggregations [37]. Mixed tubes with tunable composition and related features such as charge [38] and sizes [39] can sometimes be prepared. The unconventional amphiphilic molecular structure of BSs dictates also remarkable abilities as carriers across tissues and membranes of many compounds (e.g. drugs, carbohydrates and ions) $[40,41]$. Therefore, they are often employed as encapsulators, dispersants and carriers in complex systems $[9,42]$. Mixtures of bile salts and derivatives with thermoresponsive block copolymers have been recently investigated for their potential in these applications [43-46].

On the other side, phospholipids are amphiphiles that constitute the major component of cell membranes. Lecithin, is a typical subclass of phospholipids. It is a two-tails surfactant and has a zwitterionic polar head as it contains a positively charged choline moiety and a negative phosphate group (Fig. 1). Lecithin is known to self-assemble in water in form of pile of bilayers (lamellar phase) that in proper conditions can lead to the formation of closed vesicles (liposomes). By contrast, when added alone to suitable organic solvents (oil), it assembles into spherical or ellipsoidal reverse micelles [47]. When a polar solvent

\footnotetext{
* Corresponding author.

E-mail address: luciano.galantini@uniroma1.it (L. Galantini).
} 
<smiles>CCCCC/C=C\C/C=C\CCCCCCCC(=O)OCC(COP(=O)(O)OCC[N+](C)(C)C)OC(=O)CCCCCCCCC</smiles>

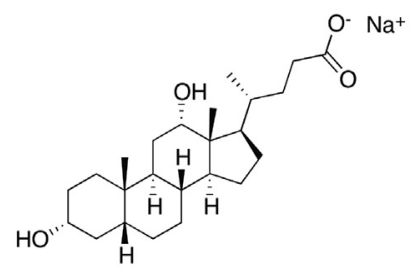

$\mathrm{NaDC}$

Fig. 1. Molecular structure of the bile salt sodium deoxycholate $(\mathrm{NaDC})$ and Lecithin.

such as water is added to these solutions in small concentrations, the micelles grow axially into flexible cylinders (for recent reviews see Refs. [48-50]). The growth of these cylinders and their subsequent entanglements into a transient network transform the sample from a lowviscosity solution into a viscoelastic one. In turn, the zero-shear viscosity is enhanced by several orders of magnitude relative to that of neat lecithin solutions and a transition from Newtonian to nonNewtonian behavior is observed (mostly depending on the nature of the oil). Beside water, bile salts have been shown to have an analogous effect of primer of the mono-dimensional growth of lecithin reverse micelles [51,52]. Bile salts are insoluble in oils but become soluble in the presence of lecithin due to intermolecular hydrogen bonds between bile salts and lecithin. In this way bile salts can induce the formation of cylindrical reverse micellar aggregates, thus playing a role similar to that of water. The molar ratio of bile salt or water to lecithin is the key parameter in dictating reverse micellar growth. In addition to water and bile salts several other polar additives have been reported to induce reverse wormlike micellar growth in dispersions of lecithin in oil [48]. In general, the additives must be not soluble in oils and strongly interact with lecithin, to work as promoter of reverse wormlike micelle growth. However, it is not completely clear how the additives interact and are arranged in the reverse micelles. It is expected that the added molecules form hydrogen bonds with lecithin and modify the packing parameter of the amphiphile to values suitable for the formation of cylindrical aggregates [51,52].

The ability of water and bile salts, as separate primers of the wormlike reverse micelle growth has been very well characterized, in recent works (see [48] and references therein). However, to our knowledge, no data have been reported so far on the growth of wormlike micelles in the presence of both of these primers. In order to provide some insight into such growth, the association properties in dispersions of lecithin, water and the bile salt sodium deoxycholate $(\mathrm{NaDC})$ in oil have been investigated. By systematically changing the fraction of the two additives we tried first to give a rough description of the phase behavior of the mixture. Therefore, a structural analysis of the aggregates was performed by combining rheology and small angle $\mathrm{X}$-ray scattering (SAXS) techniques. The results allowed us to further clarify the interaction mechanism of lecithin and primers.

\section{Material and methods}

\subsection{Materials}

Soybean lecithin (Avanti Polar Lipids 95\% purity) containing $97.2 \mathrm{wt} \%$ phosphatidylcholine and $2.8 \mathrm{wt} \%$ lysophosphatidylcholine was used. Sodium deoxycholate ( $>99 \%$ purity) containing 1.5 water molecules per mole of NaDC, Cyclohexane (>99.5\%) containing $100 \mathrm{ppm}$ of water and methanol (>99.5\%) were purchased from
Sigma-Aldrich.

\subsection{Sample preparation}

As NaDC is insoluble in cyclohexane, lecithin/NaDC mixtures were first prepared in methanol, a common solvent for both, according to the procedure proposed by Raghavan and coworkers [51]. NaDC and lecithin were separately solubilized in methanol to obtain $0.2 \mathrm{M}$ stock solutions. Then, samples at the desired compositions were prepared by mixing proper volumes of the two stock solutions. Afterwards, methanol was removed by drying under vacuum for $48 \mathrm{~h}$, and solid mixtures of lecithin and bile salt were formed. To obtain the final samples at the desired concentrations cyclohexane was added to the solid mixtures. The solution was then stirred until it became transparent and homogeneous. The procedure also ensured the elimination of removable residual water from the sample. Samples were equilibrated for at least 3 days before measurements. The composition was expressed as molar ratio of $\mathrm{NaDC}\left(\mathrm{B}_{0}\right)$ and water $\left(\mathrm{W}_{0}\right)$ with respect to lecithin, defined as $\mathrm{B}_{0}=[\mathrm{NaDC}] /[$ lecithin $]$ and $\mathrm{W}_{0}=\left[\mathrm{H}_{2} \mathrm{O}\right] /$ [lecithin $]$.

\subsection{Oscillatory rheology}

Oscillatory rheology experiments were performed on an AR1000 stress-controlled rheometer (TA Instruments). A cone-and-plate geometry $\left(40 \mathrm{~mm}\right.$ diameter $/ 4^{\circ}$ cone angle) was used. The plates were equipped with a Peltier-based temperature control, and all samples were studied at $20 \pm 0.1^{\circ} \mathrm{C}$. A solvent trap was used to minimize solvent evaporation. Oscillatory experiments in stress sweep mode at a fixed frequency of $1.0 \mathrm{~Hz}$ were first performed. These curves allowed the estimation of the linear viscoelastic domain (LVD), where Hooke's law holds and the elastic modulus is independent of the applied stress. Frequency sweep measurements at a constant applied stress amplitude falling in the LVD were collected, and rheograms of the elastic G' and viscous G" moduli as a function of an ascending frequency ramp were reported.

\subsection{SAXS measurements}

SAXS measurements were performed at the MAX II SAXS beamline I911-4 at MAX IV Laboratory in Lund, Sweden [53]. Calibration measurements were carried out using a $\mathrm{LaB}_{6}$ sample. The solutions were injected into thermostated quartz capillary sample holders and equilibrated for at least $20 \mathrm{~min}$ before measurement. The scattering intensity was recorded at $\lambda=0.91 \AA$ on a $165 \mathrm{~mm}$ diameter PILATUS $1 \mathrm{M}$ detector. The two-dimensional (2D) SAXS patterns were processed using the Fit2D software. Scattering curves were recorded within the range of $0.1<q<4.0 \mathrm{~nm}^{-1}$, where $q=(4 \pi \sin \theta) / \lambda$ ( $\theta$ being half the scattering angle). The curves were corrected for solvent and capillary contributions.

The Indirect Fourier Transform method, developed in the ATSAS program [54], was used for interpreting the SAXS curves. With this method, the pair-distance distribution functions of the single scattering particle $p(r)$ or of the particle cross section $p_{c s}(r)$ (in the case of rod-like particles) are extracted by indirect Fourier transform of the scattered intensity $I(q)$ or of the $q I(q)$ profile. The pair-distance distribution functions $p(r)$ adopts the value of zero at distances greater than the maximum size of the particle $D_{m}$ and permits the determination of its electronic gyration radius $R_{g}$ as

$R_{g}=\left[\frac{\int_{0}^{\infty} r^{2} p(r) d r}{2 \int_{0}^{\infty} p(r) d r}\right]^{\frac{1}{2}}$

Similarly, the maximum size $D_{m c}$ and the gyration radius $R_{g c}$ of the cross section can be estimated from the pair-distance distribution function $p_{c s}(r)$ of rods. 
Modeling of SAXS data was conducted using the form factor of homogeneous ellipsoids and rigid or flexible cylinders [55-57]. By assuming negligible interparticle interactions we considered $I(q)=k P(q)$ where $P(q)$ is the micelle form factor and contrast and concentration effects are included in a common scale constant $k$. The form factor depends on the scattering particle geometry, namely on the semimajor and semiminor axes ( $a_{e}$ and $b_{e}$ respectively) for ellipsoids or on length $L$ and cross section diameter $d$ for rigid cylinders. The form factor expressions for these geometries have been carefully described and extensively used to interpret the SAXS curves of bile salt micelles $[58,59]$.

For flexible cylinders, the form factor for a wormlike chain with excluded-volume interactions as derived in the paper by Pedersen and Schurtenberger [57] and then modified by Chen et al. [56], was used. The Kuhn length $b$, describing the local stiffness of the flexible cylinder, together with the contour length $L$ and the cross section diameter $d$ are needed to describe the model. All these parameters are included in the expression of the form factor and were varied in the fitting procedures together with the scale constant $k$.

\section{Results and discussion}

\subsection{Phase map}

Different mixtures of lecithin and NaDC were prepared as reported in the experimental section at a constant concentration of lecithin of $100 \mathrm{mM}$. After the equilibration time the vials were turned upside down to visually inspect their viscosity. Starting from a very low viscous solution of lecithin we observed that the viscosity increased gradually with the bile salt molar ratio $B_{0}$. We could observe the viscosity increase up to the maximum molar ratio $\mathrm{B}_{0, \max }=0.45$. At higher $\mathrm{B}_{0}$ values a phase separation was observed between a denser phase on the bottom of the vial containing both lecithin and bile salt and an upper phase that was a diluted solutions of reverse micelles formed by the exceeding surfactants [51]. On the other hand, when the lecithin/cyclohexane solution was loaded with water above a critical water/lecithin molar ratio $\left(\mathrm{W}_{0, \max }\right)$ it phase separated according to the classical Winsor II phase equilibrium (i.e. reverse micellar solution above pure excess water) $[60,61]$. In the present work, lecithin/NaDC/water microemulsions in cyclohexane were prepared starting from pre-equilibrated lecithin/NaDC solutions by adding suitable amounts of water. A common trend was found, namely that the lower the $\mathrm{B}_{0}$ the higher was the amount of water that could be stably dissolved before the phase separation took place. Accordingly, for $\mathrm{B}_{0}=0.1$ (lowest $\mathrm{B}_{0}$ value explored) the $\mathrm{W}_{0, \text { max }}$ was 11 while for the solutions at $\mathrm{B}_{0, \max }=0.45$ separation was observed already upon addition of the first water aliquot (corresponding to $\mathrm{W}_{0}=3$ ). The results were summarized in the phase map of Fig. 2. We emphasize that for the samples containing bile salts the observed phase separation was between a stiff phase on the bottom in equilibrium with an upper phase that was a diluted solutions of reverse micelles

\subsection{Oscillatory rheology}

Oscillatory rheology data were collected for representative samples at different $\mathrm{B}_{0}$ and $\mathrm{W}_{0}$ values and at constant lecithin concentration of $100 \mathrm{mM}$ and were shown in Fig. 3. The oscillatory rheology of ternary lecithin organogels upon addition of either water either BS was studied in detail $[51,52,62]$.

The reverse micelles in the system lecithin/water/cyclohexane are known to be truly wormlike, meaning that there are no signs of micellar branching, as it has previously been demonstrated by NMR selfdiffusion experiments [50]. In such disconnected micelles, micelle recombination reactions only involve micelle ends, the concentration of which are very low when the micelles are very long. Hence, the typical $\mathrm{G}^{\prime}-\mathrm{G}^{\prime \prime}$ crossover frequency is $\leq 0.1 \mathrm{rad} / \mathrm{s}$ with a corresponding

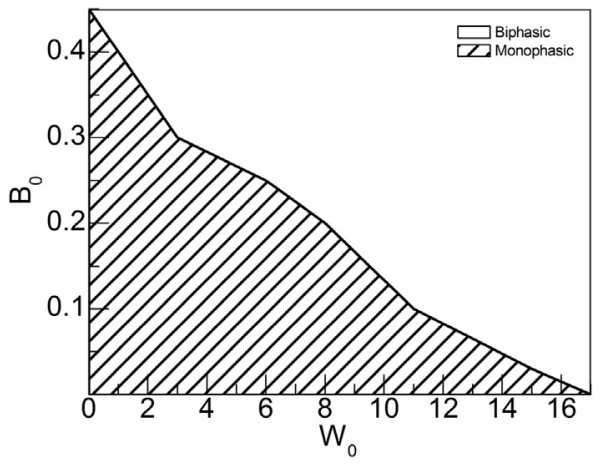

Fig. 2. Phase map of Lecithin/NaDC/water mixtures in cyclohexane at constant lecithin concentration of $100 \mathrm{mM}$ and as a function of the $\mathrm{NaDC}$ and water molar ratios $\mathrm{B}_{0}$ and $\mathrm{W}_{0}$, respectively. The area marked with parallel lines represents the region of homogeneous samples, the border line was drawn to mark the one phase region by joining the compositions at highest ratios of the primers before phase separation.

relaxation time $t_{R} \geq 10 \mathrm{~s}$, confirming that the break and recombination kinetics are very slow for these true wormlike micelles [63]. In addition, previous studies performed using frequency sweep in the range of $0.1 \mathrm{rad} / \mathrm{s}-100 \mathrm{rad} / \mathrm{s}$ have demonstrated that their rheological behavior strongly deviates from that of single Maxwell's elements - they behave as a solution of conventional (unbreakable) polymers [64]. Due to instrumental limits, we were able to probe only high frequency oscillations (between 1 and $100 \mathrm{rad} / \mathrm{s}$ ). Accordingly, for all the samples without bile salt $\left(\mathrm{W}_{0}=6,8,11,13\right)$ only the region of the rubberlike viscoelastic behavior where $G^{\prime}$ is larger than $G^{\prime \prime}$ could be assayed.

At variance with respect to the lecithin/water/cyclohexane systems, in the case of BS/lecithin/cyclohexane organogels the viscoelastic response is reported to be described by a single Maxwell's element with a single characteristic time in the range 1-0.05 s ( $\mathrm{G}^{\prime}-\mathrm{G}^{\prime \prime}$ crossover between 1 and $20 \mathrm{rad} / \mathrm{s}$ ) depending on the $\mathrm{B}_{0}$ and on the nature of the bile salt $[51,52]$. The comparison of our data on the samples at $B_{0}$ values of 0.3 and 0.4 suggests that a crossover shift to lower frequencies is induced progressively by increasing the bile salt fraction.

By contrast, starting from the lecithin/water/cyclohexane mixtures the rheology data demonstrate that the addition of bile salt induces in general an increase of the crossover frequencies. The effect is very poor at the lowest $\mathrm{NaDC}$ fractions $\left(\mathrm{B}_{0}=0.1\right)$ where, at all the measured $\mathrm{W}_{0}$ values, the samples are still characterized by a very long characteristic time (the $\mathrm{G}^{\prime}-\mathrm{G}^{\prime \prime}$ crossover frequencies below $1 \mathrm{rad} / \mathrm{s}$ ) and a behavior similar to that observed for the samples with no bile salt. The effect become instead relevant at $\mathrm{B}_{0}=0.2$. At this NaDC molar ratio we observe in particular that the crossover points move in the range $1-10 \mathrm{rad} / \mathrm{s}$ for the sample at $\mathrm{W}_{0}=3$ and to values $>100 \mathrm{rad} / \mathrm{s}$ at $\mathrm{W}_{0}=6$, thus indicating a more efficient acceleration of the dynamics of the system at higher water molar ratios.

A Maxwell behavior is indeed observed, for the samples with $\mathrm{B}_{0}=0.4$ and $\mathrm{W}_{0}=0$ and $\mathrm{B}_{0}=0.2$ and $\mathrm{W}_{0}=3$. These samples show a viscous behavior, with $G^{\prime \prime}$ exceeding $G^{\prime}$ at low frequencies or long time scales. By contrast, the samples show an inversion of the modulus at high $\omega$ or short time scales suggesting a high frequency elastic behavior. According to the Maxwell model the following equations can describe the moduli $G^{\prime}(\omega)=\frac{G_{p} \omega^{2} t_{R}^{2}}{1+\omega^{2} t_{R}^{2}}, G^{\prime \prime}(\omega)=\frac{G_{p} \omega t_{R}}{1+\omega^{2} t_{R}^{2}}$, where, $G_{p}$ is the plateau modulus, i.e., the value of $\mathrm{G}^{\prime}$ in the high-frequency limit. As shown in Fig. 4, the Maxwell model fits the data reasonably well, especially at low and intermediate frequencies, which confirms that a single relaxation time (or a narrow spectrum of relaxation times) dominates the rheological response of this sample. Maxwell fluidlike behavior is indicative of entangled wormlike micelles having a breaking time much shorter than the time of stress relaxation. The dominant relaxation time $t_{\mathrm{R}}$ of these viscoelastic samples can be estimated as $1 /$ $\omega_{c}$, where $\omega_{c}$ is the frequency at which $\mathrm{G}^{\prime}$ and $\mathrm{G}^{\prime \prime}$ cross. The estimated $t_{\mathrm{R}}$ values are around $0.14 \mathrm{~s}$ and $0.17 \mathrm{~s}$ for the samples at $\mathrm{B}_{0} / \mathrm{W}_{0}$ of $0.40 / 0$ 

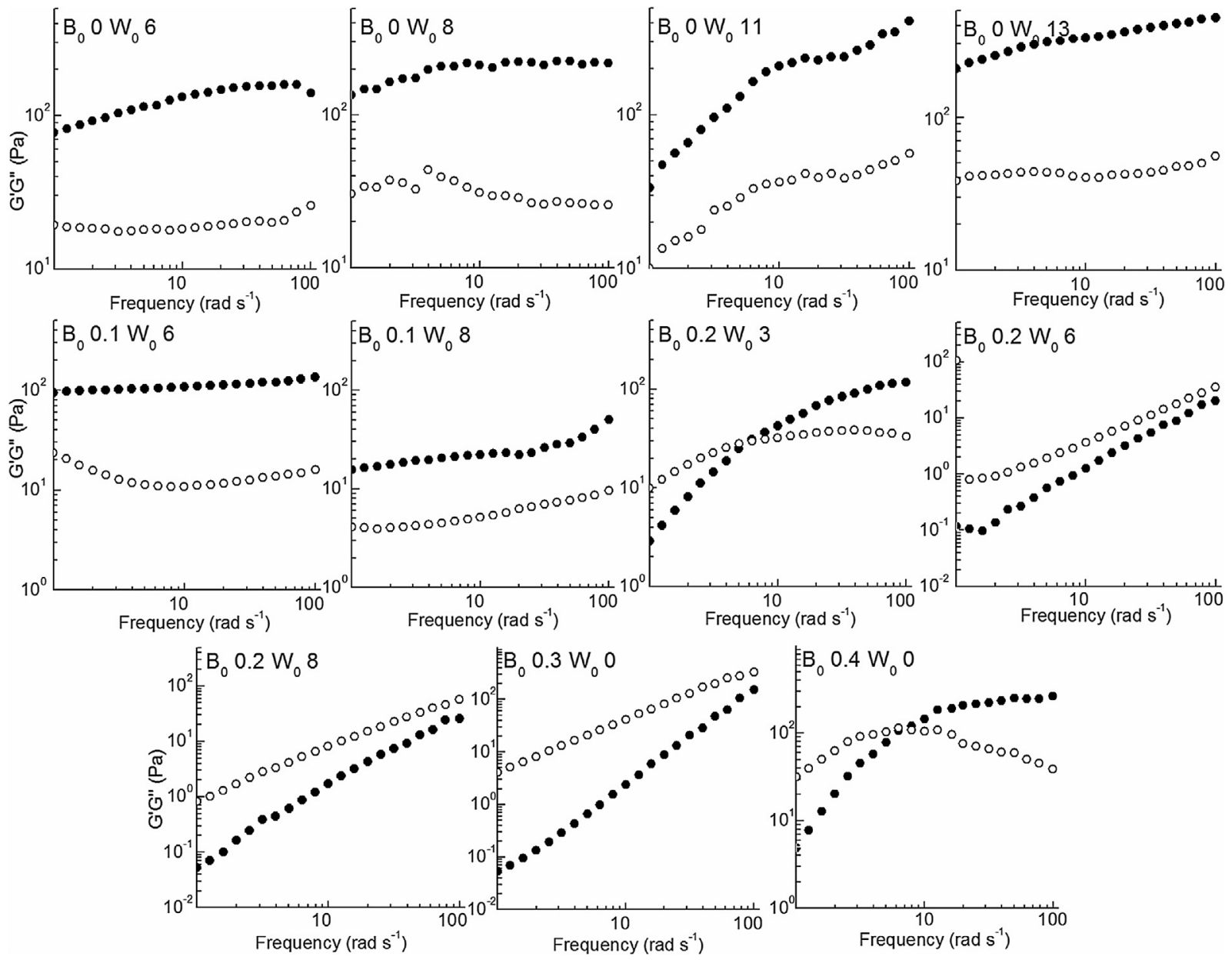

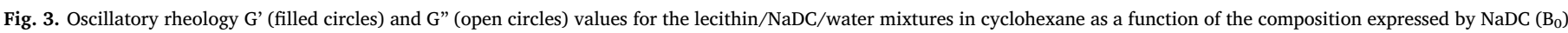
and water $\left(\mathrm{W}_{0}\right)$ molar fractions.
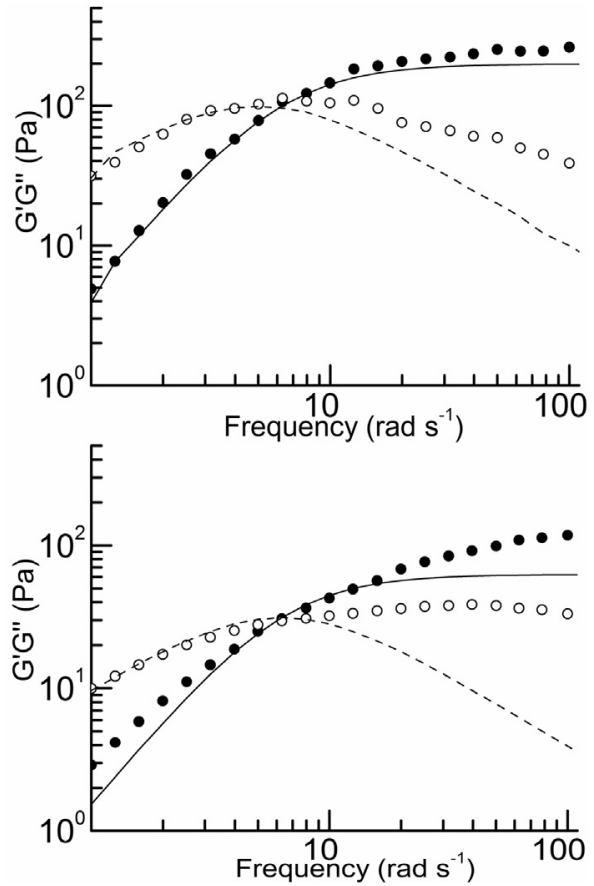

Fig. 4. Oscillatory rheology G' (filled circles) and G" (open circles) values and fits based on the Maxwell model for the mixtures in cyclohexane lecithin/NaDC at $\mathrm{B}_{0}=0.4$ (a) and lecithin/ $\mathrm{NaDC} /$ water at $\mathrm{B}_{0}=0.2$ and $\mathrm{W}_{0}=3(\mathrm{~b})$. and $0.2 / 3$, respectively. The former value agrees with the one reported in literature for lecithin/NaDC mixtures in cyclohexane at the same NaDC molar ratio [51].

According to extensive literature on the rheology of wormlike micelles behaving as living polymers, in the semidilute regime, the stress is expected to relax due to a combination of reptation and micellar breaking, with time scales $\tau_{r e p}$ and $\tau_{b r}$, respectively. In the "living" regime, when $\tau_{b r}<<\tau_{\text {rep }}$, the viscoelastic behavior is essentially Maxwell-like with a single relaxation time, $t_{R}$, given by the geometric mean $t_{R}=\left(\tau_{b r} \tau_{\text {rep }}\right)^{1 / 2}$ [65]. If $\tau_{b r}<<\tau_{\text {rep }}$, as implied by the Maxwell behavior, this implies in turn that, for $t_{R} \sim 0.17 \mathrm{~s}, \tau_{b r}<<1 \mathrm{~s}$. However, the reverse process, recombination, needs to occur on the same time scale as breaking. Recombination through the diffusion controlled fusion process of micellar ends is indeed expected to be slow ( $\tau_{b r}>>1 \mathrm{~s}$ ), because in the case of giant wormlike micelles, the micelle diffusion is low and also the concentration of micellar ends is very low [63].

According to this line of thought, in the case of wormlike micelles containing NaDC and water where $t_{R}$ is on the order of a second or less, the recombination process is not restricted to the fusion of ends only but allows also the fusion of one end with another micelle all along its contour length. But, if this is a frequent process we rather have a living network of branched micelles than a solution of living polymers, with a rheology that depends on the branching density. This could account for the decrease in viscoelastic moduli upon addition of bile salts to water/ lecithin micelles (branches can lead to a decrease in viscosity because the cross-link points can slide freely along the micellar contour) [66] and also for the peculiar phase separation found above the maximum 


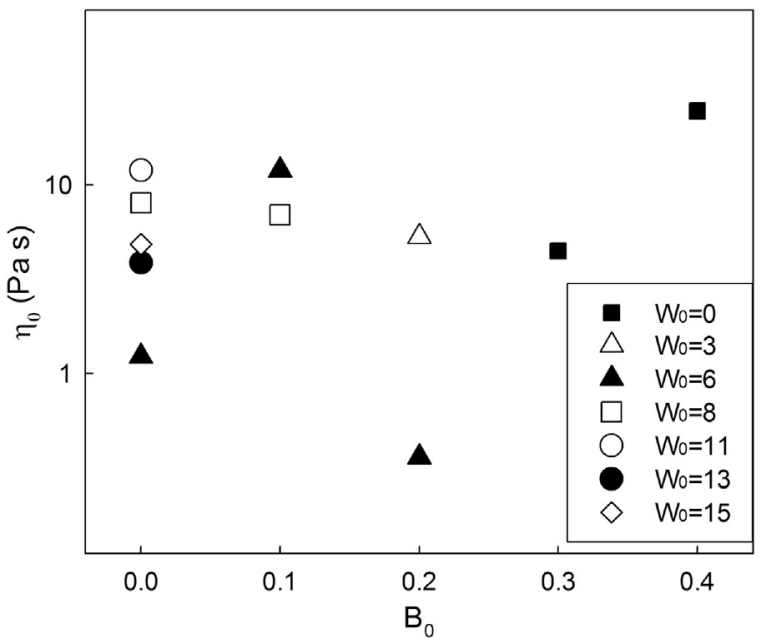

Fig. 5. Zero-shear viscosity data of lecithin/water/NaDC in cyclohexane mixtures as a function of $\mathrm{NaDC}$ molar ratio $\mathrm{B}_{0}$ for different water molar ratios $\mathrm{W}_{0}$ at $25{ }^{\circ} \mathrm{C}$.

solubilization consistent with a compact fully-branched network that collapses expelling almost pure solvent. The comparison between data at $\mathrm{B}_{0}=0.2 / \mathrm{W}_{0}=3$ and $\mathrm{B}_{0}=0.2 / \mathrm{W}_{0}=6$ indicates that the addition of water at constant bile salt ratio induces a transition from viscoelastic to viscous fluid. This means that the efficiency of bile salt to induce the formation of branches is higher the higher the water molar fraction. In other terms at constant $\mathrm{B}_{0}$ formation of branches is promoted by increasing the water content.

The zero-shear viscosity data $\left(\eta_{0}\right)$ were reported for the samples of Fig. 3 in Fig. 5 . As clearly shown by the data at $\mathrm{W}_{0}=6$, starting from the samples at $\mathrm{B}_{0}=0$, an increase of the viscosity can be appreciated after the initial additions of bile salt $\left(B_{0}=0.1\right)$, whereas a decrease is revealed at higher bile salt fraction $\left(B_{0}=0.2\right)$. This result suggests that the initial NaDC addition dictates a growth of the wormlike micelles which is reflected in the increase of the viscosity. The effect related to the formation of branches, which is also induced by the bile salt component, prevails at higher $\mathrm{B}_{0}$ values, thus justifying the viscosity lowering.

\subsection{SAXS}

SAXS experiments were performed on lecithin/bile salt/water solutions to illustrate the structure of the micelles. Solutions at lecithin concentration $20 \mathrm{mM}$ were analyzed to minimize the intermicellar interaction effects on the scattering data. The curves are plotted in Fig. 6, together with the best fitting curves and the related $p(r)$ functions obtained by the IFT method. A first analysis of the data shows that at low $\mathrm{B}_{0}$ and $\mathrm{W}_{0}$ values (sample at $\mathrm{B}_{0}=0.1, \mathrm{~W}_{0}=0$ ) the curves follow a power law behavior close to $\mathrm{I} \propto q^{\circ}$ at intermediate $q$ values, which is the scaling relationship expected for globular micelles. Accordingly, the IFT interpretation of the curves lead to a $p(r)$ curve with a symmetric bell-shaped profile. The scaling law changed to $\mathrm{I} \propto q^{-1}$ in the presence of large fractions of the two primers suggesting the formation of cylindrical aggregates. The IFT analysis provides in this case an asymmetrically shaped $p(r)$ curves, with an inflection point followed by a tail that gradually decreased to zero, which is typical of elongated aggregates. Interestingly, the ability of the primer to induce unidirectional growth of cylindrical aggregates is preserved both in the single primer (lecithin/NaDC solutions) and in the two primer samples. In the latter case the growth can be promoted equivalently by increasing the fraction of bile salt at fixed water content and vice versa by increasing the water content at fixed bile salt fraction.

It is interesting to note that a linear decrease of the $p(r)$ is observed for the samples with relatively short $D_{m}$ values (samples at $\mathrm{B}_{0} / \mathrm{W}_{0}$ of $0.1 / 3,0.1 / 6,0.2 / 3,0.3 / 0$ ) where short cylinders are formed. Shoulders at $r$ around $20-30 \mathrm{~nm}$ are instead revealed in the $p(r)$ curves with high $D_{m}$ values. This behavior is possibly due to the flexible nature of the micelles, as suggested in the literature. It must be stressed, that in the samples of the latter case the estimated $D_{m}$ values are slightly larger than the maximum dimension $\pi / q_{\min }$ ( $q_{\min }$ being the minimum accessible $q$ value of the spectrum) affordable by SAXS and therefore they cannot be considered reliable in an absolute scale. We believe however that they can be considered on a relative scale to give an idea of the wormlike micelle growth.

The IFT analysis of the $q I(q)$ vs $q$ curves was performed for the samples containing elongated micelles to elucidate their cross-section structure (Fig. 6). With this method, $p_{c s}(r)$ curves were extracted that show a bell profile not perfectly symmetric because of the presence of a little tail at high $r$ values. This profile suggests that the micelle have a cross section with a roughly circular shape (Fig. 7). The estimated radii of gyration $R_{g c}$ and maximum distance $D_{m c}$ of the cross section fall in the ranges $0.97-1.75 \mathrm{~nm}$ and $3.0-6.0 \mathrm{~nm}$, respectively (Table 1). The scattering of X-rays is provided by electrons. This means the SAXS contrast is given by the electron density difference between scatteres and their surroundings. According to a previous work, the electron density difference between the hydrophilic headgroups with bound bile salts and the hydrophobic hydrocarbon tails of lecithin dictates the contrast in lecithin/bile salt mixtures in cyclohexane. Our results suggest that a similar model of the SAXS contrast can be kept in the lecithin/water/NaDC mixtures, thus indicating that the wormlike hydrophilic core formed by lecithin headgroups, water and NaDC molecules constitute the X-ray scatterer. According to the $D_{m c}$ and $R_{g c}$ values of Table 1 the cross section of the wormlike micelles as determined by SAXS slightly expands when the fraction of water and/ or bile salt is increased in the mixture. This suggests that an expansion of the hydrophilic core takes place. SANS measurements on lecithin/ water mixtures in deuterated isooctane reported in the literature demonstrate that an increase of the cross section with water fraction occurs [67]. As both the hydrophilic core and the hydrophobic shell are expected to contribute to the SANS scattering contrast, the observed increase must be related to the whole micelle cross section. In agreement with this result, we believe that an increase of the whole micelle cross section takes place in our system in parallel with that of the hydrophilic core revealed by SAXS data.

A careful inspection of Fig. 7 allows us to appreciate that the $p_{c s}(r)$ slightly change its shape in the samples at the high water molar ratio probably reflecting changes in the electron density distribution within the SAXS revealed cross section of the micelles. The program DECON [68], based on the so-called Convolution Square Root method [69,70] and widely used to perform the deconvolution of the pair distance distribution function into radial electron density contrast profile $\Delta \rho(\mathrm{r})$ [71-73] was employed to highlight the electron density variation from the $p_{c s}(r)$ curves of Fig. 7. The obtained $\Delta \rho(\mathrm{r})$ profiles (Fig. S1) seems to show that, at a constant $B_{0}$ value, the increase of water molar ratio tends to induce an increase of the electron density in the outer region of the scattering core.

Fitting procedures were also performed by using suitable form factor models (Fig. S2). For the sample at the lowest primer fractions $\left(\mathrm{B}_{0}=0.1\right.$ and $\left.\mathrm{W}_{0}=0\right)$ where globular micelles in the early stages of the growth were expected, we observed that the curve for prolate ellipsoidal particles with semimajor and semiminor axes $a_{e}=2.29 \pm 0.02$ and $b_{e}=1.48 \pm 0.02 \mathrm{~nm}$, respectively, were able to reproduce the experimental data. For larger primer fractions fitting scattering functions for more elongated particles such as rigid or flexible cylinders were needed. Based on the IFT $p(r)$ shapes we used rigid cylinder function for the sample at intermediate primer fractions (samples at $\mathrm{B}_{0} / \mathrm{W}_{0}$ of $0.1 / 3.0,0.1 / 6.0,0.2 / 3.0$ and $0.3 / 0.0$ ). The best fitting parameters are reported in Table 2. An inspection to this Table shows that the cylinder cross section diameters $d$ obtained with this method are sensibly shorter than the $D_{m c}$ values inferred by IFT. This is not astonishing as, considering the polydispersity, the best fitting 

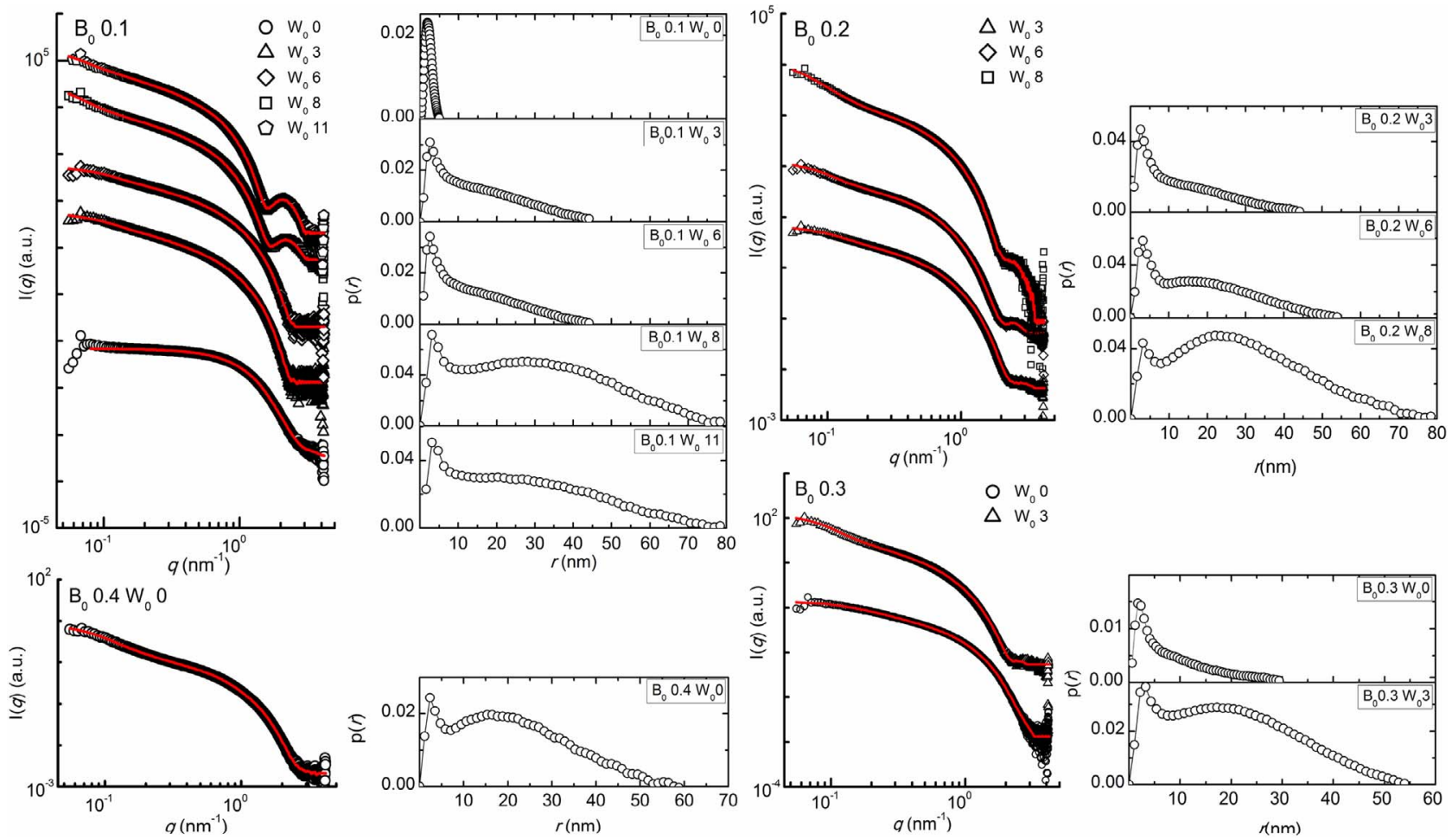

Fig. 6. SAXS experimental (symbols) and IFT is referred to the web version of this article.)
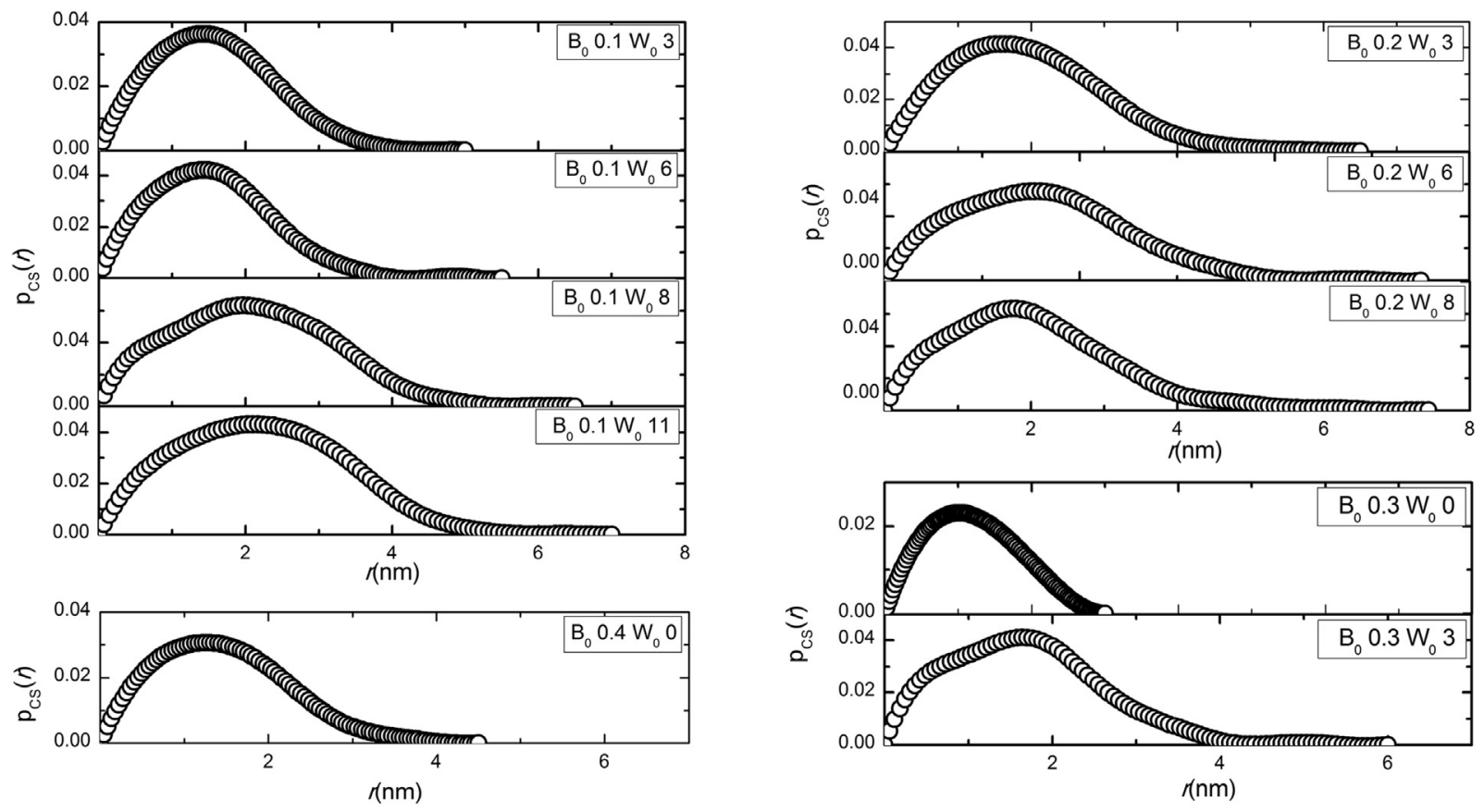

Fig. 7. Pair distribution functions of the cross section $p_{c s}(r)$ extracted by the IFT of the $q I(q)$ SAXS curves.

diameters obtained by using the model represent average values whereas the IFT $D_{m c}$ is in principle the maximum diameter of the distribution. In any case a similar trend for the $d$ and $D_{m c}$ is observed as a function of the mixture composition. Under this assumption of rigid cylinders, lengths $L$ around $45 \mathrm{~nm}$ were obtained for the lecithin/ $\mathrm{NaDC} /$ water solutions, whereas a shorter value of about $20 \mathrm{~nm}$ was inferred for the lecithin/NaDC mixture. Starting from these values a fit of the remaining samples containing very long worms was attempted by using a model for flexible chains as shown in Fig. S1. Based on the lengths obtained for rigid cylindrical micelles, values of the Kuhn length $b>45$ and $>20 \mathrm{~nm}$ were chosen for the solutions in the presence $(b=60 \mathrm{~nm})$ and in the absence $(b=30 \mathrm{~nm})$ of water. The latter was chosen in agreement with the value of $33 \mathrm{~nm}$ reported for the fit of SAXS data on wormlike micelles in NaDC/lecithin mixtures in cyclohexane with the same model [52]. The fits were performed by fixing the Kuhn length at the chosen values and by varying all the other parameters. The best fitting results are reported in Table 2 .

It must be stressed that the application of the flexible cylinder model to our SAXS data has the following limitations: i) the model does not consider that a larger cross section than the one determining the 
Table 1

Gyration radius $R_{g c}$ and maximum distance $D_{m c}$ of the cross section of the wormlike micelles inferred by analysis of the SAXS data by the IFT method.

\begin{tabular}{|c|c|c|c|}
\hline \multicolumn{2}{|c|}{ Sample } & \multicolumn{2}{|c|}{ SAXS parameters ${ }^{\mathrm{a}}$} \\
\hline $\mathrm{B}_{0}$ & $\mathrm{~W}_{0}$ & $R_{g c}(\mathrm{~nm})$ & $D_{m c}(\mathrm{~nm})$ \\
\hline 0.10 & 3.0 & 1.26 & 4.0 \\
\hline 0.10 & 6.0 & 1.39 & 4.0 \\
\hline 0.10 & 8.0 & 1.67 & 5.5 \\
\hline 0.10 & 11.0 & 1.75 & 5.5 \\
\hline 0.20 & 3.0 & 1.28 & 4.0 \\
\hline 0.20 & 6.0 & 1.53 & 5.5 \\
\hline 0.20 & 8.0 & 1.72 & 6.0 \\
\hline 0.30 & 0.0 & 0.97 & 3.0 \\
\hline 0.30 & 3.0 & 1.33 & 4.0 \\
\hline 0.40 & 0.0 & 1.17 & 4.0 \\
\hline
\end{tabular}

${ }^{\text {a }}$ The estimated uncertainties are within $0.06 \mathrm{~nm}\left(R_{g c}\right)$ and $0.4 \mathrm{~nm}\left(D_{m c}\right)$.

Table 2

Best fitting parameters length or contour length $(L)$, Kuhn length $(b)$ and cross section diameter $(d)$ obtained in the interpretation of the SAXS data on the basis of rigid or flexible cylinders.

\begin{tabular}{|c|c|c|c|c|}
\hline \multicolumn{2}{|c|}{ Sample } & \multicolumn{3}{|c|}{ SAXS parameters ${ }^{\mathrm{a}}$} \\
\hline $\mathrm{B}_{0}$ & $\mathrm{~W}_{0}$ & $b^{\mathrm{b}}(\mathrm{nm})$ & $L^{\mathrm{c}}(\mathrm{nm})$ & $d(\mathrm{~nm})$ \\
\hline 0.10 & 3.0 & & 45.2 & 3.04 \\
\hline 0.10 & 6.0 & & 45.6 & 2.94 \\
\hline 0.10 & 8.0 & 60 & 75.0 & 4.30 \\
\hline 0.10 & 11.0 & 60 & 80.8 & 4.44 \\
\hline 0.20 & 3.0 & & 45.0 & 3.04 \\
\hline 0.20 & 6.0 & 60 & 50.0 & 3.58 \\
\hline 0.20 & 8.0 & 60 & 200 & 3.70 \\
\hline 0.30 & 0.0 & & 20.8 & 2.32 \\
\hline 0.30 & 3.0 & 60 & 100 & 3.26 \\
\hline 0.40 & 0.0 & 35 & 70.2 & 2.68 \\
\hline
\end{tabular}

${ }^{a}$ The estimated uncertainties are within $0.2 \mathrm{~nm}$ (length for rigid cylinders), $10 \mathrm{~nm}$ (contour length for flexible cylinders) and $0.03 \mathrm{~nm}(d)$.

${ }^{\mathrm{b}}$ No value is reported for rigid cylinder model, values for flexible cylinders are fixed in the fit.

c Simply length for rigid cylinder model.

scattering contrast should be considered to estimate the excluded volume effect on the flexible cylinder form factor; ii) best fitting contour length were obtained for the worms that are significantly beyond the maximum dimension accessible by the scattering data. These limitations arise questions on the reliability of the best fitting parameters obtained by the model. However we observed that, considering the polydispersity effect, the best fitting cross section diameters were in reasonable agreement with the $D_{m c}$ values obtained by the IFT method. Moreover, they followed the same general growing trend by increasing the water content at fixed $\mathrm{B}_{0}$ and vice-versa the $\mathrm{NaDC}$ molar ratio at a fixed $\mathrm{W}_{0}$ value.

It is important to remark that the branches, which are supposed to induce the viscoelastic to viscous behavior transition in the samples at lecithin concentration of $100 \mathrm{mM}$, are probably not relevantly formed at the SAXS lecithin concentration $(20 \mathrm{mM})$ where worms significantly shorter than those analyzed by rheology are expected to form. Moreover, it is generally accepted that static scattering can detect micellar growth but is insufficient to determine branching [74]. On the other hand, the SAXS data presented here clearly show that wormlike micelles with a similar cross section are formed in all the analyzed samples. This confirm that branches, rather than the formation of reverse micelles with different shape, dictates the transition of the sample rheology behavior from viscoelastic to viscous, by increasing water at constant bile salt fraction or vice versa.

\section{Conclusions}

Starting from the knowledge that water and bile salts can separately act as primers to promote the spherical to wormlike transition of lecithin reverse micelles in oil, we investigated here the effect of mixtures of the two primers on the reverse micelles of lecithin in cyclohexane. In the absence of water we could reproduce the transition in the lecithin/NaDC systems and the gradual unidirectional growth of the wormlike micelles by increasing the NaDC fraction up to a phase separation occurring at NaDC molar ratio $>0.45$. Therefore, by starting from the lecithin/NaDC mixtures, an analogous growth was observed by increasing water concentration. Phase separation was observed in these mixtures at water fractions that were lower the higher the NaDC fraction, thereby a triangular region at low molar ratios of the two additives was identified where stable wormlike micelle dispersions were formed. Oscillatory rheology measurements showed that in the lecithin/water/bile salt system at constant water molar ratio the increase of bile salt fraction dictates an increase of the G' - G" crossover frequency. Such a phenomenon has been correlated with the formation of branched wormlike micelles. Therefore, we could conclude that the ability of bile salt and water to transform the originally spherical lecithin reverse micelles into wormlike micelles and thereby impart to the sample viscoelastic properties was preserved in the three solute mixtures. The results suggest that reverse micelle including both bile salt and water are formed in these systems. As already hypothesized for the solutions containing the two primers separately, reasonably water and NaDC interact with the same region of the lecithin headgroups and are complementary in altering the packing parameter of the amphiphile to values suitable to the formation of cylindrical aggregates. SAXS measurements allowed for the investigation of the cross section of the hydrophilic core of the wormlike micelles including lecithin headgroups, water and bile salt. The analysis suggested that some inhomogeneity in the electron density contrast distribution is induced by the inclusion of water in the core. It is important to clarify that a complementary interaction do not strictly implies that alternatively water or NaDC bind a headgroup. More reasonably hydrated NaDC molecules are expected to interact with the lecithin head. Accordingly, a comprehensive packing model should include interaction between $\mathrm{NaDC}$ and water molecules. However, our results did not allow us to provide details on these interactions.

\section{Acknowledgements}

The authors acknowledge MAX-IV for beam time and EU for financial support by 7th Framework Program CALIPSO (ID20140464). This work benefitted from SasView software, originally developed by the DANSE project under NSF award DMR-0520547.

\section{Appendix A. Supplementary data}

Supplementary data associated with this article can be found, in the online version, at http://dx.doi.org/10.1016/j.colsurfa.2017.04.052.

\section{References}

[1] A.S. Luk, E.W. Kaler, S.P. Lee, Structural mechanisms of bile salt-induced growth of small unilamellar cholesterol-lecithin vesicles, Biochemistry 36 (1997) 5633-5644, http://dx.doi.org/10.1021/bi962332f.

[2] L. Arleth, R. Bauer, L.H. Øgendal, S.U. Egelhaaf, P. Schurtenberger, J.S. Pedersen, Growth behavior of mixed wormlike micelles: a small-angle scattering study of the lecithin-bile salt system, Langmuir 19 (2003) 4096-4104, http://dx.doi.org/10. 1021/la026808+.

[3] M.A. Long, E.W. Kaler, S.P. Lee, G.D. Wignall, Characterization of lecithintaurodeoxycholate mixed micelles using small-angle neutron scattering and static and dynamic light scattering, J. Phys. Chem. 98 (1994) 4402-4410, http://dx.doi. org/10.1021/j100067a031.

[4] C.Y. Cheng, H. Oh, T.Y. Wang, S.R. Raghavan, S.H. Tung, Mixtures of lecithin and bile salt can form highly viscous wormlike micellar solutions in water, Langmuir 30 (2014) 10221-10230, http://dx.doi.org/10.1021/la502380q.

[5] A.F. Hofmann, D.M. Small, Detergent properties of bile salts: correlation with physiological function, Annu. Rev. Med. 18 (1967) 333-376, http://dx.doi.org/10. 1146/annurev.me.18.020167.002001.

[6] A. Sarkar, A. Ye, H. Singh, On the role of bile salts in the digestion of emulsified 
lipids, Food Hydrocoll. 60 (2016) 77-84, http://dx.doi.org/10.1016/j.foodhyd. 2016.03.018.

[7] L. Galantini, N.V. Pavel, Collective diffusion and self-diffusion coefficients comparison to separate interactions and micellar size effects on ionic micelle diffusivities: cylindrical micelles of sodium taurodeoxycholate, J. Chem. Phys. 118 (2003) 2865-2872, http://dx.doi.org/10.1063/1.1536050.

[8] A. Bonincontro, A.A. D'Archivio, L. Galantini, E. Giglio, F. Punzo, X-ray, electrolytic conductance, and dielectric studies of bile salt micellar aggregates, Langmuir 16 (2000) 10436-10443, http://dx.doi.org/10.1021/la000844w.

[9] L. Galantini, M.C. di Gregorio, M. Gubitosi, L. Travaglini, J. Vázquez Tato, A. Jover, F. Meijide, V.H. Soto Tellini, N.V. Pavel, Bile salts and derivatives: rigid unconventional amphiphiles as dispersants, carriers and superstructure building blocks, Curr. Opin. Colloid Interface Sci. 20 (2015) 170-182, http://dx.doi.org/10.1016/j cocis. 2015.08.004.

[10] D. Madenci, S.U. Egelhaaf, Self-assembly in aqueous bile salt solutions, Curr. Opin. Colloid Interface Sci. 15 (2010) 109-115, http://dx.doi.org/10.1016/j.cocis.2009. 11.010 .

[11] A.A. D'Archivio, L. Galantini, E. Gavuzzo, E. Giglio, L. Scaramuzza, Possible models for the micellar aggregates of glycocholate and taurocholate salts from crystal structures, QELS, and CD measurements, Langmuir 7463 (1996) 4660-4667.

[12] A. Bonincontro, G. Briganti, A.A. D'Archivio, L. Galantini, E. Giglio, Structural study of the micellar aggregates of sodium taurodeoxycholate, J. Phys. Chem. B 101 (1997) 10303-10309.

[13] M. Gomez-Mendoza, M.L. Marin, M.A. Miranda, Photoactive bile salts with critical micellar concentration in the micromolar range, Phys. Chem. Chem. Phys. 18 (2016) 12976-12982, http://dx.doi.org/10.1039/C6CP00813E.

[14] M. Gomez-Mendoza, M.L. Marin, M.A. Miranda, Two-channel dansyl/tryptophan emitters with a cholic acid bridge as reporters for local hydrophobicity within supramolecular systems based on bile salts, Org. Biomol. Chem. 12 (2014) 8499-8504, http://dx.doi.org/10.1039/C4OB01394H.

[15] L. Travaglini, M. Gubitosi, M.C. di Gregorio, A. D’Annibale, F. Meijide, M. Giustini, S. Sennato, M. Obiols-Rabasa, K. Schillén, N.V. Pavel, L. Galantini, A tryptophansubstituted cholic acid: expanding the family of labelled biomolecules, Colloids Surfaces A Physicochem. Eng. Asp. 483 (2015) 142-149, http://dx.doi.org/10. 1016/j.colsurfa.2015.03.033.

[16] M.C. Di Gregorio, N.V. Pavel, J. Miragaya, A. Jover, F. Meijide, J. Vázquez Tato, V.H. Soto Tellini, L. Galantini, Catanionic gels based on cholic acid derivatives, Langmuir 29 (2013) 12342-12351, http://dx.doi.org/10.1021/la402602d.

[17] A. Chakrabarty, U. Maitra, A.D. Das, Metal cholate hydrogels: versatile supramolecular systems for nanoparticle embedded soft hybrid materials, J. Mater. Chem. 22 (2012) 18268, http://dx.doi.org/10.1039/c2jm34016j.

[18] H. Svobodová, V. Noponen, E. Kolehmainen, E. Sievänen, Recent advances in steroidal supramolecular gels, RSC Adv. 2 (2012) 4985, http://dx.doi.org/10.1039/ c2ra01343f.

[19] P. Terech, N.M. Sangeetha, U. Maitra, Molecular hydrogels from bile acid analogues with neutral side chains: network architectures and viscoelastic properties. Junction zones, spherulites, and crystallites: phenomenological aspects of the gel metastability, J. Phys. Chem. B 110 (2006) 15224-15233, http://dx.doi.org/10.1021/ jp060425t.

[20] P. Terech, N.M. Sangeetha, B. Demé, U. Maitra, Self-assembled networks of ribbons in molecular hydrogels of cationic deoxycholic acid analogues, J. Phys. Chem. B 109 (2005) 12270-12276, http://dx.doi.org/10.1021/jp050666l.

[21] G. Li, Y. Hu, J. Sui, A. Song, J. Hao, Hydrogelation and crystallization of sodium deoxycholate controlled by organic acids, Langmuir 32 (2016) 1502-1509, http:// dx.doi.org/10.1021/acs.langmuir.6b00019.

[22] G. Li, Y. Wang, L. Wang, A. Song, J. Hao, Hydrogels of superlong helices to synthesize hybrid Ag-Helical nanomaterials, Langmuir 32 (2016) 12100-12109, http://dx.doi.org/10.1021/acs.langmuir.6b03052.

[23] M. Zhang, S. Strandman, K.C. Waldron, X.X. Zhu, Supramolecular hydrogelation with bile acid derivatives: structures, properties and applications, J. Mater. Chem. B 4 (2016) 7506-7520, http://dx.doi.org/10.1039/C6TB02270G.

[24] U. Maitra, M. Maity, Supramolecular gels from bile acid-amino acid conjugates and their applications, Eur. J. Org. Chem. 2017 (13) (2017) 1713-1720, http://dx.doi. org/10.1002/ejoc.201601616.

[25] T.T.T. Myllymäki, H. Yang, V. Liljeström, M.A. Kostiainen, J.M. Malho, X.X. Zhu, O. Ikkala, Hydrogen bonding asymmetric star-shape derivative of bile acid leads to supramolecular fibrillar aggregates that wrap into micrometer spheres, Soft Matter. 12 (2016) 7159-7165, http://dx.doi.org/10.1039/c6sm01329e.

[26] S. Ikonen, A. Valkonen, R. Juvonen, H. Salo, E. Kolehmainen, Bile acid-derived mono-and diketals-synthesis, structural characterization and self-assembling properties, Org. Biomol. Chem. 8 (2010) 2784-2794, http://dx.doi.org/10.1039/ c003228j.

[27] L. Travaglini, A. D’Annibale, M.C. Di Gregorio, K. Schillén, U. Olsson, S. Sennato, N.V. Pavel, L. Galantini, Between peptides and bile acids: self-assembly of phenylalanine substituted cholic acids, J. Phys. Chem. B 117 (2013) 9248-9257, http://dx.doi.org/10.1021/jp405342v.

[28] L. Travaglini, A. D’Annibale, K. Schillén, U. Olsson, S. Sennato, N.V. Pavel, L. Galantini, Amino acid-bile acid based molecules: extremely narrow surfactant nanotubes formed by a phenylalanine-substituted cholic acid, Chem. Commun. 48 (2012) 12011-12013, http://dx.doi.org/10.1039/c2cc36030f.

[29] L. Travaglini, M. Gubitosi, M.C. di Gregorio, N.V. Pavel, A. D’Annibale, M. Giustini, V.H. Soto Tellini, J. Vázquez Tato, M. Obiols-Rabasa, S. Bayati, L. Galantini, On the self-assembly of a tryptophan labeled deoxycholic acid, Phys. Chem. Chem. Phys. 16 (2014) 19492-19504, http://dx.doi.org/10.1039/c4cp02371d.

[30] M. Gubitosi, L. Travaglini, A. D’Annibale, N.V. Pavel, J. Vázquez Tato, M. ObiolsRabasa, S. Sennato, U. Olsson, K. Schillén, L. Galantini, Sugar-bile acid-based bolaamphiphiles: from scrolls to monodisperse single-walled tubules, Langmuir 30 (2014) 6358-6366, http://dx.doi.org/10.1021/la500908r.

[31] M. Gubitosi, A. D’Annibale, K. Schillén, U. Olsson, N.V. Pavel, L. Galantini, On the stability of lithocholate derivative supramolecular tubules, RSC Adv. 7 (2017) 512-517, http://dx.doi.org/10.1039/C6RA26092F.

[32] V.H. Soto Tellini, A. Jover, F. Meijide, J. Vázquez Tato, L. Galantini, N.V. Pavel, Supramolecular structures generated by a p-tert-butylphenylamide derivative of cholic acid: from vesicles to molecular tubes, Adv. Mater. 19 (2007) 1752-1756, http://dx.doi.org/10.1002/adma.200602581.

[33] B. Jean, L. Oss-Ronen, P. Terech, Y. Talmon, Monodisperse bile-salt nanotubes in water: kinetics of formation, Adv. Mater. 17 (2005) 728-731, http://dx.doi.org/10. 1002/adma.200401125.

[34] P. Terech, A. De Geyer, B. Struth, Y. Talmon, Self-assembled monodisperse steroid nanotubes in water, Adv. Mater. 14 (2002) 495-498, http://dx.doi.org/10.1002/ 1521-4095(20020404)14:7<495:AID-ADMA495 > 3.0.CO;2-9.

[35] M.C. di Gregorio, N.V. Pavel, A. Jover, F. Meijide, J. Vázquez Tato, V.H. Soto Tellini, A.A. Vargas, O. Regev, Y. Kasavi, K. Schillén, L. Galantini, pH sensitive tubules of a bile acid derivative: a tubule opening by release of wall leaves, Phys. Chem. Chem. Phys. 15 (2013) 7560-7566, http://dx.doi.org/10.1039/c3cp00121k.

[36] M.C. di Gregorio, M. Varenik, M. Gubitosi, L. Travaglini, N.V. Pavel, A. Jover, F. Meijide, O. Regev, L. Galantini, Multi stimuli response of a single surfactant presenting a rich self-assembly behavior, RSC Adv. 5 (2015) 37800-37806, http:// dx.doi.org/10.1039/C5RA01394A.

[37] L. Galantini, C. Leggio, A. Jover, F. Meijide, N.V. Pavel, V.H. Soto Tellini, J. Vázquez Tato, R. Di Leonardo, G. Ruocco, Kinetics of formation of supramolecular tubules of a sodium cholate derivative, Soft Matter. 5 (2009) 3018-3025, http://dx.doi.org/10.1039/b905531b.

[38] N. Manghisi, C. Leggio, A. Jover, F. Meijide, N.V. Pavel, V.H. Soto Tellini, J. Vázquez Tato, R.G. Agostino, L. Galantini, Catanionic tubules with tunable charge, Angew. Chem. Int. Ed. 49 (2010) 6604-6607, http://dx.doi.org/10.1002/ anie.201000951.

[39] M. Gubitosi, L. Travaglini, M.C. Di Gregorio, N.V. Pavel, J. Vázquez Tato, S. Sennato, U. Olsson, K. Schillén, L. Galantini, Tailoring supramolecular nanotubes by bile salt based surfactant mixtures, Angew. Chem. Int. Ed. 54 (2015) 7018-7021, http://dx.doi.org/10.1002/anie.201500445.

[40] M. Stojančević, N. Pavlović, S. Goločorbin-Kon, M. Mikov, Application of bile acids in drug formulation and delivery, Front. Life Sci. 7 (2013) 112-122, http://dx.doi. org/10.1080/21553769.2013.879925.

[41] W. Kramer, Transporters, Trojan horses and therapeutics: suitability of bile acid and peptide transporters for drug delivery, Biol. Chem. 392 (2011) 77-94, http://dx. doi.org/10.1515/bc.2011.017.

[42] M. Gubitosi, J.V. Trillo, A. Alfaro Vargas, N.V. Pavel, D. Gazzoli, S. Sennato, A. Jover, F. Meijide, L. Galantini, Characterization of carbon nanotube dispersions in solutions of bile salts and derivatives containing aromatic substituents, J. Phys. Chem. B. 118 (2014) 1012-1021, http://dx.doi.org/10.1021/jp407145t.

[43] S. Bayati, C. Anderberg Haglund, N.V. Pavel, L. Galantini, K. Schillén, Interaction between bile salt sodium glycodeoxycholate and PEO-PPO-PEO triblock copolymers in aqueous solution, RSC Adv. 6 (2016) 69313-69325, http://dx.doi.org/10. 1039/C6RA12514J.

[44] S. Bayati, L. Galantini, K.D. Knudsen, K. Schillén, Effects of bile salt sodium glycodeoxycholate on the self-assembly of PEO-PPO-PEO triblock copolymer P123 in aqueous solution, Langmuir 31 (2015) 13519-13527, http://dx.doi.org/10. 1021/acs.langmuir.5b03828.

[45] S. Bayati, L. Galantini, K.D. Knudsen, K. Schillén, Complexes of PEO-PPO-PEO triblock copolymer P123 and bile salt sodium glycodeoxycholate in aqueous solution: a small angle X-ray and neutron scattering investigation, Colloids Surf. A Physicochem. Eng. Asp. 504 (2016) 426-436, http://dx.doi.org/10.1016/j.colsurfa. 2016.05.096.

[46] M.C. di Gregorio, M. Gubitosi, L. Travaglini, N.V. Pavel, A. Jover, F. Meijide, J. Vázquez Tato, S. Sennato, K. Schillén, F. Tranchini, S. De Santis, G. Masci, L. Galantini, Supramolecular assembly of a thermoresponsive steroidal surfactant with an oppositely charged thermoresponsive block copolymer, Phys. Chem. Chem. Phys. 19 (2017) 1504-1515, http://dx.doi.org/10.1039/C6CP05665B.

[47] P. Walde, A.M. Giuliani, C.A. Boicelli, P.L. Luisi, Phospholipid-based reverse micelles, Chem. Phys. Lipids 53 (1990) 265-288, http://dx.doi.org/10.1016/00093084(90)90026-N.

[48] G. Palazzo, Wormlike reverse micelles, Soft Matter. 9 (2013) 10668-10677, http:// dx.doi.org/10.1039/c3sm52193a.

[49] R. Angelico, S. Murgia, G. Palazzo, Wormlike micelles. Advances in systems, characterisation and applications, in: Y.F.C.A. Dreiss (Ed.), Wormlike Micelles Adv. Syst. Characterisation Appl. The Royal Society of Chemistry, Cambridge, UK, 2017.

[50] R. Angelico, U. Olsson, G. Palazzo, A. Ceglie, Surfactant curvilinear diffusion in giant wormlike micelles, Phys. Rev. Lett. 81 (1998) 2823-2826, http://dx.doi.org/ 10.1103/PhysRevLett.81.2823.

[51] S.-H. Tung, Y.-E. Huang, S.R. Raghavan, A new reverse wormlike micellar system: mixtures of bile salt and lecithin in organic liquids, J. Am. Chem. Soc. 128 (2006) 5751-5756, http://dx.doi.org/10.1021/JA0583766.

[52] C.-W. Njauw, C.-Y. Cheng, V.A. Ivanov, A.R. Khokhlov, S.-H. Tung, Molecular interactions between lecithin and bile salts/acids in oils and their effects on reverse micellization, Langmuir 29 (2013) 3879-3888, http://dx.doi.org/10.1021/ la304601p.

[53] A. Labrador, Y. Cerenius, C. Svensson, K. Theodor, T. Plivelic, The yellow minihutch for SAXS experiments at MAX IV laboratory, J. Phys. Conf. Ser. 425 (2013) 072019, http://dx.doi.org/10.1088/1742-6596/425/7/072019.

[54] M.V. Petoukhov, D. Franke, A.V. Shkumatov, G. Tria, A.G. Kikhney, M. Gajda, C. Gorba, H.D.T. Mertens, P.V. Konarev, D.I. Svergun, IUCr, new developments in 
the ATSAS program package for small-angle scattering data analysis, J. Appl Crystallogr. 45 (2012) 342-350, http://dx.doi.org/10.1107/S0021889812007662.

[55] J.S. Pedersen, Analysis of small-angle scattering data from colloids and polymer solutions: modeling and least-squares fitting, Adv. Colloid Interface Sci. 70 (1997) 171-210, http://dx.doi.org/10.1016/S0001-8686(97)00312-6.

[56] W.-R. Chen, A. Paul, D. Butler, Linda J. Magid, Incorporating intermicellar interactions in the fitting of SANS data from cationic wormlike micelles, Langmuir 22 (2006) 6539-6548, http://dx.doi.org/10.1021/LA0530440.

[57] J.S. Pedersen, P. Schurtenberger, Scattering functions of semiflexible polymers with and without excluded volume effects, Macromolecules 29 (1996) 7602-7612, http://dx.doi.org/10.1021/ma9607630.

[58] S. Cozzolino, L. Galantini, C. Leggio, N.V. Pavel, Correlation between small-angle Xray scattering spectra and apparent diffusion coefficients in the study of structure and interaction of sodium taurodeoxycholate micelles, J. Phys. Chem. B 109 (2005) 6111-6120, http://dx.doi.org/10.1021/jp044540k.

[59] L. Galantini, E. Giglio, A. Leonelli, N.V. Pavel, An integrated study of small-angle Xray scattering and dynamic light scattering on cylindrical micelles of sodium glycodeoxycholate, J. Phys. Chem. B 108 (2004) 3078-3085, http://dx.doi.org/10. 1021/jp036746b.

[60] R. Angelico, A. Ceglie, U. Olsson, G. Palazzo, Phase diagram and phase properties of the system lecithin-water-cyclohexane, Langmuir 16 (2000) 2124-2132, http://dx. doi.org/10.1021/LA9909190.

[61] R. Angelico, G. Palazzo, G. Colafemmina, P.A. Cirkel, M. Giustini, A. Ceglie, Water diffusion and headgroup mobility in polymer-like reverse micelles: evidence of a sphere-to-rod-to-sphere transition, J. Phys. Chem. B 102 (1998) 2883-2889, http:// dx.doi.org/10.1021/JP980194T.

[62] S.-H. Tung, S.R. Raghavan, Strain-stiffening response in transient networks formed by reverse wormlike micelles, Langmuir 24 (2008) 8405-8408, http://dx.doi.org/ 10.1021/la704045t.

[63] U. Olsson, J. Börjesson, R. Angelico, A. Ceglie, G. Palazzo, Slow dynamics of wormlike micelles, Soft Matter. 6 (2010) 1769-1777, http://dx.doi.org/10.1039/ b920115g.

[64] R. Angelico, S. Amin, M. Monduzzi, S. Murgia, U. Olsson, G. Palazzo, Impact of branching on the viscoelasticity of wormlike reverse micelles, Soft Matter. 8 (2012) 10941-10949, http://dx.doi.org/10.1039/c2sm26528a.

[65] M.E. Cates, Reptation of living polymers: dynamics of entangled polymers in the presence of reversible chain-scission reactions, Macromolecules 20 (1987) 2289-2296, http://dx.doi.org/10.1021/ma00175a038.

[66] T.J. Drye, M.E. Cates, Living networks: the role of cross-links in entangled surfactant solutions, J. Chem. Phys. 96 (1992) 1367-1375, http://dx.doi.org/10. 1063/1.462172.

[67] G. Jerke, J. Pedersen, S. Egelhaaf, P. Schurtenberger, Static structure factor of polymerlike micelles: overall dimension, flexibility, and local properties of lecithin reverse micelles in deuterated isooctane, Phys. Rev. E 56 (1997) 5772-5788, http://dx.doi.org/10.1103/PhysRevE.56.5772.

[68] R. Mittelbach, O. Glatter, Direct structure analysis of small-angle scattering data from polydisperse colloidal particles, J. Appl. Crystallogr. 31 (1998) 600-608, http://dx.doi.org/10.1107/S0021889898002209.

[69] O. Glatter, Convolution square root of band-limited symmetrical functions and its application to small-angle scattering data, J. Appl. Crystallogr. 14 (1981) 101-108, http://dx.doi.org/10.1107/S002188988100887X.

[70] O. Glatter, Comparison of two different methods for direct structure analysis from small-angle scattering data, J. Appl. Crystallogr. 21 (1988) 886-890, http://dx.doi. org/10.1107/S0021889888007381.

[71] M. Tomšič, M. Bešter-Rogač, A. Jamnik, W. Kunz, D. Touraud, A. Bergmann, O. Glatter, Ternary systems of nonionic surfactant Brij 35, water and various simple alcohols: structural investigations by small-angle X-ray scattering and dynamic light scattering, J. Colloid Interface Sci. 294 (2006) 194-211, http://dx.doi.org/10. 1016/j.jcis.2005.06.088.

[72] M. Tomšič, M. Bešter-Rogač, A. Jamnik, W. Kunz, D. Touraud, A. Bergmann, O. Glatter, Nonionic surfactant Brij 35 in water and in various simple alcohols: structural investigations by small-angle X-ray scattering and dynamic light scattering, J. Phys. Chem. B 108 (2004) 7021-7032,, http://dx.doi.org/10.1021/ jp049941e.

[73] D. Löf, M. Tomšič, O. Glatter, G. Fritz-Popovski, K. Schillén, Structural characterization of nonionic mixed micelles formed by C 12 EO 6 surfactant and P123 triblock copolymer, J. Phys. Chem. B 113 (2009) 5478-5486, http://dx.doi.org/10. 1021/jp808442d.

[74] M.A. Calabrese, S.A. Rogers, R.P. Murphy, N.J. Wagner, The rheology and microstructure of branched micelles under shear, J. Rheol. 59 (2015) 1299-1328, http://dx.doi.org/10.1122/1.4929486. 